\title{
RELAXATION PHENOMENA IN EXCITED MOLECULES
}

\author{
Stuart A. Rice and William M. Gelbart \\ Department of Chemistry and The James Franck Institute, \\ The University of Chicago, Chicago, Illinois 60637, USA
}

\begin{abstract}
This paper reviews the interpretation of radiationless transitions, thermal reactions and photochemical rearrangements and decompositions, developed in the last three years. In each of these cases, the relaxation process involved can be described within a common theoretical framework by simply observing that the system of interest is prepared in a compound (nonstationary) state. We begin (part II) by briefly discussing the role of zero-order properties (e.g. interaction energies and densities of states) in determining the time scales appropriate to the observation of irreversible decay in isolated molecules. We then (part III) identify the various ways in which the usual classification of excited molecular states breaks down. A simple, but quite general, energy level model is shown to represent the essential features of the spectrum of zero-order vibronic manifolds in polyatomic molecules: absorption line shapes, interference effects, and emission lifetimes and quantum yields, are briefly discussed. The experimentally observed dependencies of the nonradiative transition rate on electronic energy gap, molecular geometry and frequency changes, and isotope effects, are shown to follow directly from a many-phonon description of the electronic relaxation process. We discuss further the way in which this theoretical approach provides quantitative predictions of the dependence of the radiationless lifetimes (within a given electronic state) on vibrational excitation.

In part IV we elaborate upon the general theme of this paper, namely that the details of the nuclear and electonic couplings can directly determine the rates of, and products of, reactions in which molecules are prepared with highly non-uniform distributions of internal energy. The models of photo-dissociation and isomerism treated in earlier papers are cast in terms of a resonance scattering formalism in order to emphasize the chemical dynamics common to these diverse decomposition and rearrangement processes. An alternative to the conventional theory of unimolecular reactions is briefly reviewed-the roles of nonuniform internal energy distributions and intramolecular vibrational interactions are shown to be of considerable importance in providing a priori predictions of the dissociation kinetics in large molecules. These results are illuminated by reference to recent linear chain calculations and molecular exciton descriptions. Finally, in part V, we discuss several semi-empirical and experimental methods which we feel can complement our theoretical studies by providing unambiguous information on the actual dynamics of intramolecular energy exchange and dissociation.
\end{abstract}




\section{INTRODUCTION}

That part of chemistry which deals with the reactivity of compounds and the possible changes of molecular geometry and bonding is intimately concerned with relaxation phenomena in excited molecules. This sweeping statement is obviously correct in the case when reaction is initiated by the absorption of high energy radiation. Then, as will be argued later, preparation of a molecule in a state with nonuniform distribution of energy requires that we study the various modes of internal energy redistribution before interpretation of the chemical reaction is possible. At the other extreme, namely the case of exothermic reaction without an activation energy barrier, the reaction products are usually generated with a nonequilibrium distribution of internal energy. Hence in this limit also, because the products are in excited states, it is of interest to examine the several possible pathways for molecular relaxation.

It is most common to excite a molecule under conditions such that no chemical reactions occur. For this class of excitation it is possible to observe if, and how, an initial state of the molecule evolves with time. Of the many possible molecule-preserving relaxation processes we shall be particularly interested in those classified as radiationless transitions.

Photochemical reactions, thermal reactions and radiationless transitions appear to be very different phenomena. The apparent differences are, however, more superficial than real. In every case the relaxation process involved can be interpreted within a common theoretical framework; we need only observe that the system under investigation is prepared in a compound (nonstationary) state.

This paper briefly reviews the interpretation of radiationless processes and photochemical reactions developed in the last three years. All details of the mathematical analysis are omitted so that attention can be wholly focused on the important physical ideas.

\section{KEY CONCEPTS}

How does a relaxation process take place in a microscopic system? Suppose we describe a system, initially, with some convenient zero-order Hamiltonian, usually chosen to include as many of the system's interactions as possible consistent with mathematical tractibility of the corresponding Schroedinger equation. Suppose the spectrum corresponding to the zeroorder Hamiltonian can be partitioned into two (or more) sets of states. These states are not eigenstates of the full Hamiltonian of the system, but a true eigenstate of the system can be represented as a time independent linear superposition of the zero-order states.

The coefficients in the linear superposition depend upon the nature and magnitude of the interaction terms omitted from the zero-order Hamiltonian. It often happens that one of the sets of zero-order states (the sparse part) consists of a finite number of discrete energy levels, corresponding to a small subset of the total number of degrees of freedom of the system, while the other set of zero-order states (the dense part) has a continuous or quasi-continuous spectrum and is associated with an infinite, or effectively infinite, 


\section{RELAXATION PHENOMENA IN EXCITED MOLECULES}

number of degrees of freedom. A relaxation process takes place when a compound state of the system, consisting of some superposition of zero-order discrete and continuum states, decays into the continuum $\dagger$

The states corresponding to a specific zero-order Hamiltonian provide a convenient basis for the discussion of quantum relaxation phenomena, but these states have no real physical significance. The two subsets of zero-order levels described above are usually degenerate or quasidegenerate, and therefore extensive configuration mixing is induced by the small interaction which couples the subsets of states. Properties of the total system such as absorption coefficients, relaxation times, etc., must be described in terms of the properties of compound states.

The interpretation of relaxation phenomena requires an understanding of how an initial state of the system is prepared, and of the consequent time evolution of this state. A stationary state of the system will undergo transition to other stationary states only by coupling with the radiation field, so that all transitions between stationary states are radiative in nature. However, if a system is prepared in a nonstationary state of the total molecular Hamiltonian, 'nonradiative transitions' will occur. This follows from the observation that the initial nonstationary state can be represented as a time dependent superposition of the eigenstates of the total molecular Hamiltonian, each of which is a time independent superposition of states which correspond to the sparse and dense subsets of zero-order states. In general, if one of the subsystems of zero-order states is very dense, a compound state of the system with large amplitude of a sparse subsystem component evolves so that the continuum component grows with time. On the other hand, if the densities of states in the two subsystems of zero-order states are comparable and small, the amplitude of a component zero-order state will oscillate with time.

The preceding comment alludes to the importance of the densities of states of the several subsets of states. In a finite system the time for recurrence of an initial state is of the order of magnitude of $\rho \hbar$, where $\rho$ is the density of states $^{1}$. If the time scale of interest is much shorter than $\rho \hbar$, irreversible behaviour is observed, i.e. an initial state decays into some other set of states and does not recur. If the time scale of interest is comparable to or longer than $\rho \hbar$, reversible behaviour is observed, i.e. the initial states recurs.

The density of states also enters in an important way in the description of the distribution of amplitudes which describes the linear superposition of zero-order states representing an eigenstate of the total Hamiltonian. Let the matrix element coupling the subsets of zero-order states be $v$, and consider the case where the zero-order spectrum has one sparse subset and one dense subset of states. Suppose it is possible to create, as an initial nonstationary state, a molecule excited to one level of the sparse subset of states. When $v \rho>1$ many states of the dense subset contribute to the linear combination of states representing the eigenstate of the total Hamiltonian. If these states

$\dagger$ Of course, it is also possible to represent any one (or any combination) of the zero order states as a linear superposition of the exact eigenstates of the system. In this case, however, the coefficients are time dependent. The interplay between the many time rates of change of the terms in the linear superposition defines the overall time rate of change of the nonstationary zero-order state. 
are indistinguishable, their phases interfere in a manner such that the probability of radiative decay of the molecule is modulated by an extra exponential decay with reciprocal lifetime $\tau_{n r}^{-1}=2 \pi v^{2} \rho / \hbar$, and ${ }^{1} \tau_{n r} \ll \rho \hbar$. In this case the quantum yield of fluorescence is less than unity. On the other hand when $v \rho<1$, usually because the densities of states in the two zero order subsets are sufficiently small that the average level spacing exceeds the interaction energy, only a few zero-order states from the second subset contribute to linear superposition representing an eigenstate of the total Hamiltonian. Then $\rho \hbar$ is shorter than the radiative lifetime, the probability of radiative decay of the molecule is altered but not exponentially modulated, and the quantum yield of fluorescence is unity.

The extension of the concepts described above to the case of photochemical reactions requires proper identification of the pertinent manifolds of states, and of the nature of their interaction. Although the details are very different, the key ideas are the same.

We now examine, in somewhat more detail, the consequences of exciting a molecule to a nonstationary state.

\section{ASPECTS OF THE THEORY OF RADIATIONLESS PROCESSES}

The first successful interpretation of radiationless processes in large aromatic molecules was advanced by Robinson and $\mathrm{Frosch}^{2}$. Their analysis included several of the ideas we now recognize as very important, but did not fully exploit the quantum mechanics of compound states. Furthermore, they restricted attention to the case $v \rho \gg 1$. Bixon and Jortner ${ }^{3}$, and Rhodes, Henry and Kasha ${ }^{4}$ developed, independently and at the same time, the compound state theory sketched in Section II. This theory, which has since been extensively expanded by Jortner, Berry, Freed, Rice and others, is described below.

Almost all quantum mechanical descriptions of molecules are based on the Born-Oppenheimer (BO) approximation. There are many different ways of describing the $\mathrm{BO}$ approximation. Perhaps the simplest is to say that, because the mass of the electron is so much smaller than the masses of the nuclei, the electrons may be assumed to move in a static potential field generated by the nuclei distributed over the equilibrium positions characteristic of the molecule. Then, in turn, the nuclei are assumed to oscillate about their equilibrium positions in an effective field generated by the instantaneously responsive electrons. The BO approximation is valid only if the off diagonal matrix elements of the nuclear kinetic energy operator in the basis of BO states are small compared to the separations of the BO states. For large molecules, with many vibrational degrees of freedom, the density of BO states is so large that it is often the case that the energy difference between BO states is very small relative to the off diagonal matrix elements of the nuclear kinetic energy. In these cases the eigenstates of the total Hamiltonian must be very different from BO states for an excited molecule, even when the true ground state is accurately approximated by the BOground state. In anthracene, for example, the density, $\rho$, of ${ }^{3} B_{2 u}$ vibrational levels in the energy region of the ${ }^{1} B_{2 u}$ vibrationless state is as large as $5 \times 10^{10} \mathrm{~cm}^{-1}$. The mean interaction energy, $v$, is estimated ${ }^{3}$ to be roughly $6 \times 10^{-7} \mathrm{~cm}^{-1}$, thereby implying 


\section{RELAXATION PHENOMENA IN EXCITED MOLECULES}

that $\rho v \approx 3 \times 10^{4} \gg 1$, i.e. $v \gg \varepsilon \equiv \rho^{-1}$, and there is extensive mixing of the zero-order vibronic states. It is also interesting to note ${ }^{3}$ that the recurrence time, $\hbar \rho \approx 0.25 \mathrm{sec}$, greatly exceeds the measured radiative lifetime $\tau \approx 5$ $\times 10^{-9} \mathrm{sec}$, consistent with the fact that the radiationless transition is observed to take place as an irreversible relaxation process. Other examples of the large molecule limit are given in Table 1. It is clear that the study of the photochemistry of these systems, which of necessity concerns itself with the energy region of electronically excited states, depends upon an understanding of the breakdown of the Born-Oppenheimer approximation and the details of the various non-radiative relaxation phenomena.

Table 1. Parameters descriptive of radiationless transitions in large molecules

\begin{tabular}{|c|c|c|c|c|c|}
\hline System & $\tau(\mathrm{sec})$ & $v\left(\mathrm{~cm}^{-1}\right)$ & $\rho(\mathrm{cm})$ & $v \rho$ & $\hbar \rho(\mathrm{sec})$ \\
\hline $\begin{array}{l}\text { Anthracene } \\
{ }^{1} B_{2 u} \overrightarrow{3}^{3} B_{2 u} \\
E=12000 \mathrm{~cm}^{-1}\end{array}$ & $5 \times 10^{-9}$ & $6 \times 10^{-7}$ & $5 \times 10^{10}$ & $3 \times 10^{4}$ & 0.25 \\
\hline $\begin{array}{l}\text { Naphthalene } \\
{ }^{1} B_{2 u} \overrightarrow{ }^{1} B_{3 u} \\
E=3400 \mathrm{~cm}^{-1}\end{array}$ & $10^{-12}$ & $6 \times 10^{-2}$ & $2 \times 10^{3}$ & $10^{2}$ & $10^{-8}$ \\
\hline $\begin{array}{l}\text { Naphthalene } \\
{ }^{3} B_{2 u} \rightarrow{ }^{1} A_{1 g} \\
E=20000 \mathrm{~cm}^{-1}\end{array}$ & 2 & $10^{-14}$ & $8 \times 10^{15}$ & 80 & $4 \times 10^{4}$ \\
\hline $\begin{array}{l}\text { Azulene } \\
{ }^{1} B_{1} \rightarrow^{1} A_{1} \\
E=14000 \mathrm{~cm}^{-1}\end{array}$ & $10^{-11}$ & $7 \times 10^{-5}$ & $10^{11}$ & $7 \times 10^{6}$ & 0.5 \\
\hline $\begin{array}{l}\text { Benzene } \\
{ }^{1} B_{2 u} \rightarrow{ }^{3} B_{1 u}\end{array}$ & $10^{-6}$ & $1.5 \times 10^{-5}$ & $8 \times 10^{4}$ & 1.5 & $4 \times 10^{-7}$ \\
\hline
\end{tabular}

Experimentally, these large-molecule $(\rho v \gg 1)$ processes are manifest in several ways, e.g.

(i) Resonance fluorescence is not observed when, for example, anthracene ${ }^{5}$, naphthalene ${ }^{6}$, and tetracene ${ }^{7}$ are optically excited to their second and higher singlet states at low gas pressures (such that the mean time between collisions greatly exceeds the pure radiative lifetime, $\tau_{r}$ ). The observed fluorescence resembles the emission which follows direct excitation to the first singlet, but is slightly red-shifted and diffuse.

(ii) The overall lifetime of the first excited singlets, when extrapolated to low gas pressures, are shorter than $\tau_{r}$ (calculated from the integrated absorption coefficient) by factors of two ${ }^{8}$ to four 9 .

(iii) The fluorescence quantum yield of benzene $\left(\phi_{f} \approx 0.34\right)$ is independent of vapour density at sufficiently low pressures, and part of the sample of excited molecules behaves chemically as if it were the lower triplet state ${ }^{10}$ (e.g. it isomerizes cis-butene).

(iv) In cases where an environment change is not expected to introduce (e.g. through external spin-orbit coupling, or crystal field effects, etc). any 
basic changes in the character of the electronic states, there is no conclusive evidence for believing that the radiationless transitions are sensibly influenced by coupling of the molecule to a dense medium.

Exceptions to the above generalizations, as well as a comprehensive discussion of the experimental background, have been considered by Jortner, Rice and Hochstrasser ${ }^{1}$.

Aside from neglecting the nuclear kinetic energy, the conventional theory of molecular electronic structure also uses, as an additional approximation, a spin independent Hamiltonian. The natural consequence of this approximation is the classification of all the electronic states of the molecule into pure spin manifolds, e.g. singlet, triplet, quintet, etc. Of course, the existence of spin-orbit coupling implies that pure spin states cannot be stationary states of the total Hamiltonian. However, the lifetime of a nonstationary pure spin state can be very long if the spin-orbit coupling matrix element is small and the states to which the initial state is coupled are sparsely distributed; the reverse is true if the density of states is large, or the spin-orbit coupling large. In the case of large molecules, for which the density of zero-order pure spin BO states is very large, the conventional classification of excited states according to spin multiplicity is no longer valid.

More explicitly, we write $v=\overline{v_{m r, n s}}$ where $v_{m r, n s} \equiv\left\langle\Psi_{m r}|T(Q)| \Psi_{n s}\right\rangle$ : $T(Q)=-\sum_{k}\left(\hbar^{2} / 2 M_{k}\right) \partial^{2} / \partial Q_{k}^{2}$ is the nuclear kinetic energy operator and $\Psi_{m r}=\psi_{m}(q, Q) \chi_{m r}(Q)$ and $\Psi_{n s} \equiv \psi_{n}(q, Q) \chi_{n s}(Q)$ are the wavefunctions corresponding to the $m r^{\text {th }}$ and $n s^{\text {th }}$ vibronic states. That is, $\psi_{m}(q, Q)$ is the $m^{\text {th }}$ solution to the electronic Schrodinger equation

$$
H_{e l} \psi_{m}(q, Q) \equiv\left[T(q)+U(q, Q)+H_{s}\right] \psi_{m}(q, Q) \equiv E_{m}(Q) \psi_{m}(q, Q)
$$

for fixed nuclear configuration $Q$ : here $T(q)$ is the kinetic energy of the electrons, $U(q, Q)$ is the Coulomb potential energy of the nuclei and electrons, and $H_{s}$ denotes the electron spin terms including the spin-orbit coupling $H_{s o}$. The vibrational wavefunction $\chi_{m r}(Q)$ is the $r^{\text {th }}$ solution to the equation

$$
\left[E_{m}(Q)+T(Q)\right] \chi_{m r}(Q) \equiv W_{m r} \chi_{m r}(Q) .
$$

Noting that the electronic wavefunctions are orthogonal in $q$-space one has that

$$
v_{m r, n s} \approx-\sum_{k} \frac{\hbar^{2}}{M_{k}} \int_{Q} \chi_{m r}^{*}(Q)\left\langle\psi_{m}(q, Q)\left|\frac{\partial}{\partial Q_{k}}\right| \psi_{n}(q, Q)\right\rangle \frac{\partial}{\partial Q_{k}} \chi_{n s}(Q) \mathrm{d} Q
$$

where we have simply neglected the second derivatives of the electronic wavefunctions with respect to the nuclear displacements. Differentiating (1) with respect to $Q_{k}$, multiplying by $\psi_{n}(q, Q)$ and integrating over all $q$, one finds that

$$
\begin{array}{r}
\left\langle\psi_{m}(q, Q)\left|\frac{\partial}{\partial Q_{k}}\right| \psi_{n}(q, Q)\right\rangle=\frac{\left\langle\psi_{m}(q, Q)\left|\left(\partial U / \partial Q_{k}\right)\right| \psi_{n}(q, Q)\right\rangle}{E_{m}(Q)-E_{n}(Q)} \\
\approx \frac{\left\langle\psi_{m}(q, 0)\left|\left(\partial U / \partial Q_{k}\right)_{0}\right| \psi_{n}(q, 0)\right\rangle}{E_{m}(0)-E_{n}(0)}
\end{array}
$$


In order to evaluate $v_{m r, n s}$ we treat the spin-orbit coupling, $H_{s o}$, as a perturbation to the zero-order electronic Hamiltonian $H_{e l}^{0}(0)$, where $H_{e l}^{0}(0)=T(q)$ $+U(q, Q=0)+\left(H_{s}-H_{s o}\right)$ with eigensolutions $\left\{\psi_{m}^{0}(q, 0)\right\}$ (the zero superscript denotes pure spin states). Thus we write, to first order,

$$
\psi_{m}(q, 0) \approx \psi_{m}^{0}(q, 0)+\sum_{\gamma \neq m} \frac{\left\langle\psi_{m}^{0}(q, 0)\left|H_{s o}\right| \psi_{\gamma}^{0}(q, 0)\right\rangle}{E_{m}^{0}(0)-E_{\gamma}^{0}(0)} .
$$

Then $v_{m r, n s}$ becomes

$$
\begin{aligned}
v_{m r n s} & \approx \sum_{k} F_{m r, n s}^{k} \\
& \times \frac{J_{m n}^{k(0)}+\sum_{\gamma \neq m, n}\left[k_{m \gamma}^{(0)} J_{\gamma n}^{k(0)} / E_{m}^{0}(0)-E_{\gamma}^{0}(0)+J_{m \gamma}^{k(0)} k_{\gamma n}^{(0)} / E_{n}^{0}(0)-E_{\gamma}^{0}(0)\right]}{E_{n}^{0}(0)-E_{m}^{0}(0)}
\end{aligned}
$$

where

and

$$
\begin{aligned}
J_{m n}^{k(0)} & \equiv\left\langle\psi_{m}^{0}(q, 0)\left|\left(\frac{\partial U}{\partial Q_{k}}\right)_{0}\right| \psi_{n}^{0}(q, 0)\right\rangle, \\
k_{m \gamma}^{(0)} & \equiv\left\langle\psi_{m}^{0}(q, 0)\left|H_{s o}\right| \psi_{\gamma}^{0}(q, 0)\right\rangle
\end{aligned}
$$

is the Franck Condon vibrational overlap integral. If $m$ and $n$ refer to states with the same spin multiplicity ('internal conversion') then only the leading term $J_{m}^{k(0)} /\left(E_{n}^{0}(0)-E_{m}^{0}(0)\right)$ need be retained in the electronic factor. When $J_{m n}^{k(0)}$ vanishes identically ('intersystem crossing') one must determine the terms in the sum over $\gamma$, according to appropriate spin orbit-vibronic coupling selection rules.

Having identified two of the possible forms of interaction between the zeroorder BO states of a molecule, we turn to an examination of the time evolution of a compound state initially prepared as a pure BO state.

Bixon and Jortner ${ }^{3}$ analyzed the simple model energy level spectrum shown in Figure 1. This model is intended to represent the essential features of the spectrum of zero-order BO vibronic levels of a large molecule. The BO state $\phi_{s}$ will at first be treated as the vibrationless level of a singlet manifold, but this is not a necessary restriction. A transition from the BO ground state $\phi_{o}$ to $\phi_{s}$ is allowed, with transition dipole moment $\mu_{o s}$. The manifold $\left\{\phi_{i}\right\}$ is taken to be the set of vibrational levels belonging to a BO singlet or triplet electronic state just below $\phi_{s}$. In this model transitions between $\phi_{o}$ and the $\left\{\phi_{i}\right\}$ are forbidden. When the $\left\{\phi_{i}\right\}$ belong to a triplet, spin orthogonality leads to $\mu_{o i}=0$, and this is not much changed by the small spin-orbit coupling typical of aromatic hydrocarbons. When the $\left\{\phi_{i}\right\}$ belong to a singlet, the Franck-Condon factors for excitation to a level near $\phi_{s}$ are so small that again $\mu_{o i}=0$. In general, the density of the levels $\left\{\phi_{i}\right\}$ degenerate with $\phi_{s}$ increases with the energy gap between the vibrationless levels of the two excited states, and with increasing number of vibrational degrees of freedom. 

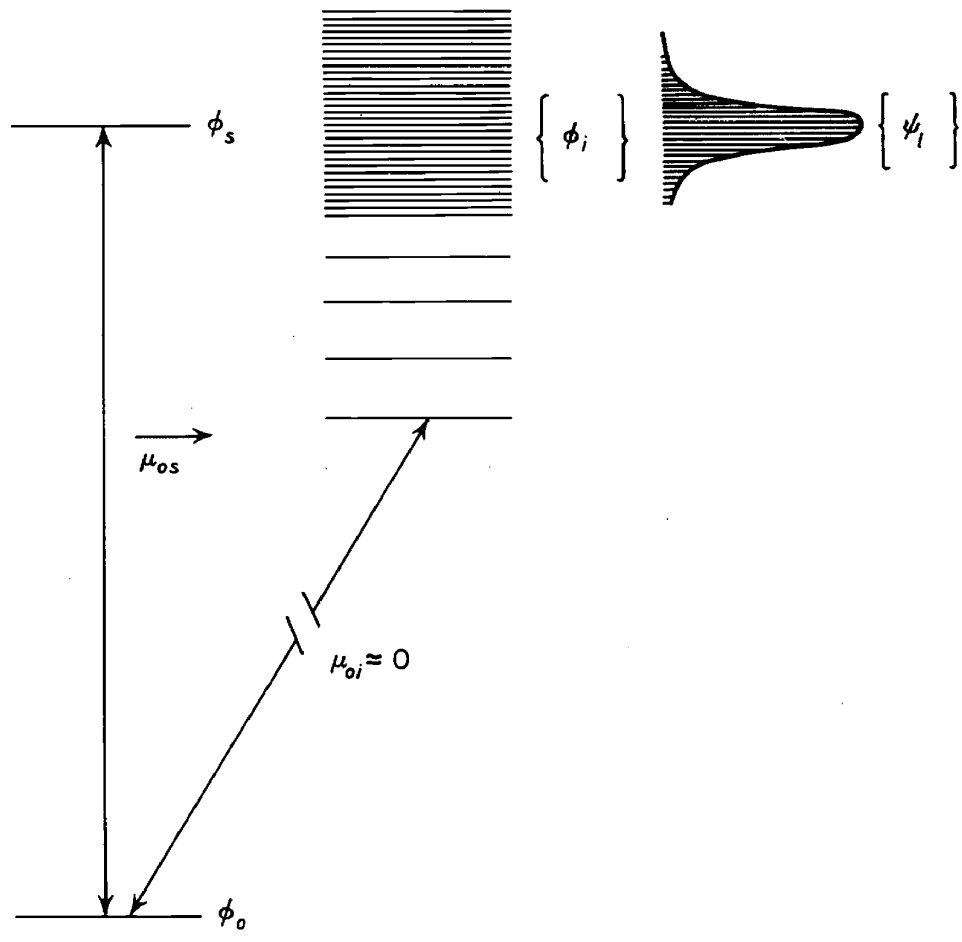

Figure 1. Energy-level scheme for a large molecule.

Because of the degeneracy, or quasi-degeneracy, of $\phi_{s}$ with a group of levels from the $\left\{\phi_{i}\right\}$, the BO representation of the excited electronic states in the model spectrum shown cannot be valid. Instead, an eigenstate of the total Hamiltonian is of the form

$$
\psi_{n}=a_{s}^{n} \phi_{s}+\sum_{i} b_{i}^{n} \phi_{i}
$$

Bixon and Jortner ${ }^{3}$ assumed that the manifold $\left\{\phi_{i}\right\}$ have uniformly spaced levels with separation $\varepsilon=\rho^{-1}$, and that the matrix element which couples $\phi_{\mathrm{s}}$ to the $\left\{\phi_{i}\right\}, v=\left\langle\phi_{s}|H| \phi_{i}\right\rangle$, is the same for all levels (hence independent of energy). It is then found that the expansion coefficients describing the weights of the zero-order state $\phi_{s}$ in the eigenstate $\psi_{n}$ is of the form

$$
\left|a_{s}^{n}\right|^{2}=\frac{v^{2}}{\left(E_{n}-E_{s}\right)^{2}+\left(\pi v^{2} \rho\right)+v^{2}}
$$

where $E_{s}$ represents the zero-order energy corresponding to $\phi_{s}$, and $E_{n}$ the eigenenergy corresponding to $\psi_{n}$. One may immediately draw four conclusions:

(i) Because of the breakdown of the $\mathrm{BO}$ approximation in the case of degenerate zero-order levels, the molecular eigenstates contain appreciable contributions from the many degenerate and quasi-degenerate zero-order 
BO states with energy close to the energy of the eigenstate. Thus. if the transition moments are such that the initial nonstationary state prepared by absorption of a photon is a pure BO state, e.g. $\phi_{s}$ of Figure 1, the absorption profile is inhomogeneously broadened. The inhomogeneous broadening results from a differential distribution of the zero-order BO component among the eigenstates of the total Hamiltonian.

(ii) The absorption line shape is Lorentzian, when $\rho v \gg 1$ :

$$
A(E) \propto \rho\left|a_{s}^{n}\right|^{2}=\frac{v^{2} \rho}{\left(E_{s}-E_{n}\right)^{2}+\left(\pi v^{2} \rho\right)^{2}} .
$$

(iii) The line width is given by

$$
\Delta=\pi v^{2} \rho .
$$

(iv) Interference effects between the discrete state and the background states occur only when the transitions to the zero-order background states carry oscillator strength. Hence, for the model spectrum of Figure 1 no Fano antiresonances ${ }^{11}$ are expected to occur.

The state mixing described above is entirely an intramolecular phenomenon; as shown it leads to the distribution of absorption intensity over a moderately large energy region. There is, however, much more that can be learned about the system by considering the mode of preparation and the time evolution of the initial compound state. Let a molecule with model spectrum as in Figure 1 be coupled to the radiation field. When the band pass of radiation incident on the molecule is such as to coherently excite the molecule (i.e. when the band pass exceeds the inhomogeneously broadened line width), there is interference among the amplitudes of the coherently excited states. Consequently, it is found that the radiationless decay of the probability amplitude of the zero-order state $\phi_{s}$ is exponential and characterized by

$$
\tau_{n r}=\hbar / 2 \pi v^{2} \rho \text {. }
$$

Thus a lifetime appears naturally (as a consequence of interference) in the description of the time evolution of the compound state and need not be introduced by ad hoc considerations, or by kinetic arguments. The transition rate is of the form of the Golden Rule of first order time dependent perturbation theory, although it does not arise from a perturbation theory analysis.

Chock, Jortner and Rice ${ }^{12}$, Berry and Jortner ${ }^{13}$, Freed and Jortner ${ }^{14}$, Bixon, Jortner and Dothan ${ }^{15}$, and Freed ${ }^{16}$ have significantly generalized the original theory of Bixon and Jortner. It is shown in these papers that a formal theory of radiationless processes, using advanced many-body techniques to describe the time evolution of the compound state and its coupling to the radiation field, leads to the same general conclusions as does the more straightforward analysis of a model spectrum of zero-order levels. These cited studies can be summarized as follows ${ }^{1}$ :

(i) A necessary condition for the occurrence of nonradiative decay of a compound state, and concomitantly of inhogeneous line broadening, is $v \rho \geqslant 1$. The nonradiative decay then occurs on a time scale short compared to $\hbar \rho$. For times longer than $\hbar \rho$, the amplitude of $\phi_{s}$ in the compound state increases towards its initial value. However, for large molecules $h \rho$ con- 
siderably exceeds the time scale on which other interfering processes occur, e.g. collision with the walls or other molecules. The density of states need not be uniform for these conclusions to be valid.

(ii) For $v \rho \gg 1$, the observed radiative lifetime, determined on a time scale short relative to $\hbar \rho$, consists of independent contributions from the nonradiative and pure radiative components. In large molecules, for which $\hbar \rho$ is much longer than any other molecular period, this result implies an exponential decay law as already noted. Exponential decay of this particular form (shortened radiative lifetime) does not occur on a time scale longer than $\hbar \rho$ (see comment (vi) below).

(iii) Let $n=\rho \Delta$ be the average number of levels within the halfwidth of the inhomogeneously broadened line. Then from $\tau_{n r}=\hbar / 2 \Delta$, we find that $\tau_{n r}=$ $\hbar \rho / 2 n$, and the lifetime against nonradiative decay can be thought of as the recurrence time reduced by the factor $2 n$.

(iv) The simple additive nonradiative decay rate $(\hbar / 2 \Delta)^{-1}$, in the overall rate of decay of a compound state coupled to the radiation field, is a valid description only if all the molecular eigenstates in an inhomogeneously broadened line correspond to a set of indistinguishable levels ${ }^{15}$. If the levels are distinguishable (e.g. Zeeman levels which emit photons of different polarization) the rate of decay of the compound state can be nonexponential under certain conditions of preparation and observation ${ }^{\mathbf{1 4}, 15}$.

(v) An excited state resulting from intramolecular radiationless transitions in a large molecule decays as follows $(t \ll \hbar \rho)$ : In the case of internal conversion from the second to the first singlet, the excited state is connected by nonvanishing radiative coupling to high vibronic levels of the ground state. In an isolated molecule, fluorescence radiation will be emitted corresponding to transitions between vibrationally excited levels of the excited and ground electronic states. As a result of this fluorescence, if the molecule stays free, it will eventually emit its excess energy as infra red radiation. However, because of the very long radiative lifetimes for vibrational transitions, relaxation by collision is also likely to take place.

Of course, corresponding to the above described decay mechanism, the quantum yield of fluorescence is less than unity; only a fraction $Y<1$ of the absorbed photons can be emitted on the time scale $t \ll \hbar \rho$. The fraction $1-Y$ will be emitted, as described above, on a time scale long compared to the fluorescence decay time.

(vi) Singlet excited states of small molecules, e.g. triatomics, are quasidegenerate with only a few vibronic states belonging to the lower triplet state and the ground state. However the coupling matrix element is, in certain cases, sufficiently large that $v \rho \geqslant 1$, so that inhomogeneous broadening of the absorption line can occur. Because the density of levels is small the recurrence time for the compound state is very short, indeed much shorter than the lifetime against fluorescence decay or the time between collisions. Although for a time $t \ll \hbar \rho$ the overall decay rate has a radiative and nonradiative component, just as for a large molecule, this is unobservable because of the short recurrence time. When $t \gg \hbar \rho$, which is a time scale on which experiments can be conducted, the radiative decay consists of a sum of exponential decays, one for each of the separate molecular eigenstates ${ }^{17}$. In this case, when the level spacing exceeds the radiative width $\Gamma$, i.e. $\rho^{-1}>\Gamma$, 
the apparent excited state lifetime becomes $n \hbar / \Gamma$, and hence is lengthened relative to the radiative lifetime expected on the basis of the integrated oscillator strength ${ }^{17}$. The conditions cited are encountered in the cases of $\mathrm{NO}_{2}$ and $\mathrm{SO}_{2}$, both of which are known to have 'anomalously long' radiative lifetimes ${ }^{19}$.

(vii) The occurrence of inhomogeneous broadening in small molecules implies the spreading out of the $\phi_{s}$ oscillator strength, and this redistribution leads to the occurrence of many new lines in the spectrum (corresponding to transitions to all the molecular eigenstates $\psi_{n}$ ). This is probably the source of the 'extra' regularly spaced lines in the spectrum of $\mathrm{NO}_{2}{ }^{18}$.

(viii) The Lorentzian absorption line shape, and all the other particulars cited above, represent the description of an isolated resonance degenerate with a single quasi-continuum. When two or more zero-order levels interact with a quasicontinuum, and the widths of the two states exceed their separation, the absorption line shape is not Lorentzian and the nonradiative decay rate is not exponential in form. When one zero-order level interacts with two or more quasicontinua, and both of the continua are sufficiently dense, the absorntion line shape is still Lorentzian, but with width determined by the sum $\sum_{i} v_{i}^{2} \rho_{i}$.

Much of the above information is displayed for convenient comparison in Table 2.

Table 2. The radiative decay of a manifold of indistinguishable levels

\begin{tabular}{|c|c|c|c|}
\hline Physical property & Resonance limit & Intermediate case & Statistical limit \\
\hline$n=\pi v^{2} \rho^{2}$ & - & $n \sim 10-100$ & $n \gg 1$ \\
\hline Line shape & $\begin{array}{l}\text { natural radiative } \\
\text { + conventional } \\
\text { broadening }\end{array}$ & $\begin{array}{l}\text { inhomogenous } \\
\text { broadening, } \\
\text { separable lines }\end{array}$ & $\Delta=\pi v^{2} \rho$ \\
\hline $\begin{array}{l}\text { Time scale relative to } \\
\text { recurrence time }(\hbar \rho)\end{array}$ & - & $t \gg \hbar \rho$ & $t \ll \hbar \rho$ \\
\hline Form of decay & beat spectrum & non exponential & exponential \\
\hline Radiative decay time & $\tau_{\text {exp }}=1 / \Gamma$ & $\tau_{\exp } \propto n / \Gamma$ & $\tau_{\mathrm{exp}}=\Gamma \Delta / \Gamma+\Delta$ \\
\hline $\begin{array}{l}\text { Fluorescence quantum } \\
\text { yield on time scale } t\end{array}$ & $Y=1$ & $Y=1$ & $Y<1$ \\
\hline Features of relaxation & external & external & intramolecular \\
\hline Examples & $\mathrm{CN}$ & $\mathrm{SO}_{2}, \mathrm{NO}_{2}, \mathrm{CS}_{2}$ & $\begin{array}{l}\text { Benzene } \\
\text { Naphthalene } \\
\text { Anthracene }\end{array}$ \\
\hline
\end{tabular}

Several years ago it was observed, semi-empirically, by Robinson and Frosch $^{2}$, Siebrand ${ }^{19}$, and Ross ${ }^{20}$, and their coworkers, that the efficiencies of nonradiative processes depend directly on the energy gap ( $0-0$ transition energy), $\Delta E$, the changes in equilibrium configurations and frequencies of the vibrations, and isotope substitution. It is interesting to note that this situation 
is largely analogous to that encountered in cases of localized electronic transitions in solids, e.g. absorption by F-centres (trapped electrons) or isolated impurities in crystals ${ }^{2}$. Using the general line shape formalism developed by Kubo ${ }^{22}$ to describe these latter multiphonon processes, Lin and Bersohn ${ }^{23}$ and Englman and Jortner ${ }^{24}$ have derived all of the experimentally observed dependences of the nonradiative transition probabilities on the electronic energy difference, molecular geometry and frequency changes, and deuterium effects. Gelbart, Freed and Rice ${ }^{25}$ have extended these approaches to take into account the necessarily large change in equilibrium configurations accompanying intramolecular rearrangements of the cis-trans type. Also, Freed and Jortner ${ }^{26}$ have explicitly treated the role of the promoting mode and have shown that, in the case of the aromatic hydrocarbons, it is possible to derive an energy gap law which correlates all the important features of the semi-empirical analyses.

At the same time these extensions of the multiphonon formulation point up the usefulness of keeping the nonradiative rate expression in the form of a constant times a sum over squares of the vibrational overlap integrals between the initial vibrationless state and the nearly degenerate vibronic levels of the lower electronic state. (Contact with the earlier treatments can be made by approximating this sum by the product of a mean Franck-Condon factor $\left(v^{2}\right)$ times the number of terms in the sum $(\rho)$ : in this way one recovers the familiar result that the rate is proportional, through the 'electronic factor' to the product $v^{2} \rho$ ). More explicitly, Freed and Jortner ${ }^{26}$ and Gelbart et al. ${ }^{27}$ show that this sum assumes the form of a microcanonical partition function for 'phonons' which obey Boltzmann statistics and which are distributed amongst states that have 'degeneracies' $\left\{x_{j} \equiv \Delta_{j}^{2} / 2\right\}$, where $\Delta_{j} \equiv\left(M_{j} \omega_{j} / \hbar\right)^{\frac{1}{2}}$ $\Delta Q_{i}^{0}$ is the reduced displacement between the two electronic states in the equilibrium position of the $j^{\text {th }}$ normal coordinate (with effective mass $M_{j}$ and frequency $\omega_{j}$ ). In other words we have that

$$
k_{n r} \propto \sum_{\left\{n_{j}\right\}} \prod_{j} \frac{x_{j}^{n_{j}}}{n_{j} !} \delta\left[\Delta E-\sum_{j} n_{j} \hbar \omega_{j}\right],
$$

where $\Delta E_{k}=\Delta E-\hbar \omega_{k}, k$ denoting the promoting mode. For large enough $\Gamma E_{k}$ this sum can be approximated by the corresponding canonical ensemble partition function; viz. $\sum_{\left\{n_{j}\right\}}\left(\prod_{j} x_{j}^{n_{j}} / n_{j} !\right)$ is evaluated subject to that constraint of conservation of the mean energy. That is, neglecting fluctuations, we have that

$$
\Delta E_{k}=\sum_{j} n_{j} \hbar \omega_{j}
$$

is the electronic energy available for distribution over the internal degrees of freedom. As is customary in the statistical mechanical analogue, the canonical ensemble sum, subject to (13), is approximated by its maximum term, using the method of Lagrange multipliers. It is found that $n_{j}^{0}$, the most probable number of quanta accepted by the $j^{\text {th }}$ vibration in the non-radiative transition, increases exponentially with the frequency $\omega_{j}$ and linearly with the displacement $x_{j}$ : this result is consistent with the semi-empirical finding that the best accepting modes are those of maximum frequency, and that among this class the most efficient are those which undergo the largest geometry change between initial and final electronic states. Substituting for $n_{j}^{0}$ in the maximum term, 


\section{RELAXATION PHENOMENA IN EXCITED MOLECULES}

$\prod_{j}\left(x_{j}^{n_{j}} / n_{j}^{0} !\right)$ and, noting that the $x_{j}$ 's are less than unity for the aromatic hydrocarbons, it is found that the most likely distribution of energy $\Delta E_{k}$ amongst the internal degrees of freedom is that which allows for the smallest values of $n_{j}^{0}$ and hence $\sum_{j} n_{j}^{0}$. This result is simply that which would be expected in an electronic transition (from the vibrationless level of the initial state) for which the Franck-Condon principle was operative. It is also shown ${ }^{27}$ that the maximum term can be written in the 'energy gap' form derived by more exact methods by Englman and Jortner.

Most importantly, Gelbart et al. ${ }^{27}$ use the maximum term method to calculate the energy dependence of the nonradiative rate constant in a molecule under 'collision-free' conditions : they determine the variation of the radiationless lifetimes of resolved vibrational levels in an excited electronic state. Earlier theoretical work had implicitly assumed that the initial state of the optically excited molecule was the vibrationless level resulting from rapid collisional deactivation. Descriptions of this kind had been sufficient to explain the variety of data obtained for molecules in solution, dense gases, and solid hosts. Recently, however, experiments have been carried out at low enough pressures that the optically excited gas molecules decay nonradiatively on a time scale much shorter than the mean time between deactivating collisions. Furthermore, with a bandwidth of, say, an Angstrom, the initial states of the excited molecule can be meaningfully described in terms of the individual normal modes.

Gelbart $e{ }{ }^{27} l^{27}$ have calculated the variation in nonradiative lifetime $\tau_{n r}$ of the benzene ${ }^{1} B_{2 u}$ electronic state, excited with 0,1 , and 2 quanta in the totally symmetric skeletal (breathing) vibration: they find that $\tau_{n r}$ decreases by factors of 0.83 and 0.70 as one populates the $v_{1}$ mode with 1 and 2 quanta. These numerical estimates are shown to be consistent with the corresponding fluorescence lifetimes $\tau$ measured at $\approx 0.1$ Torr using nanosecond flash excitation and time correlated photon counting (see Table 3). Agreement between theory and experiment can be sharpened as soon as more accurate quantum yield $\left(\phi_{f}\right)$ data become available ${ }^{28}$, thereby allowing us to separate out more cleanly the pure radiative contribution $\tau_{r}$ to the observed lifetime $\left[\tau \equiv\left\{\tau_{r} \tau_{n r} /\left(\tau_{r}+\tau_{n r}\right)\right\}=\tau_{n r}\left(1-\phi_{f}\right)\right]$

Table 3.

\begin{tabular}{ccc}
\hline Vibrational excitation in ${ }^{1} B_{2 u}$ & Observed lifetime $\tau$ (nsec) & Standard deviation (nsec) \\
\hline $1^{0}$ & 102.9 & 2.7 \\
$1^{1}$ & 80.5 & 1.4 \\
$1^{2}$ & 61.7 & 0.9 \\
$6^{1} 1^{0}$ & 81.5 & 0.4 \\
$6^{1} 1^{1}$ & 73.7 & 0.4 \\
$6^{1} 1^{2}$ & 57.0 & 0.4 \\
\hline
\end{tabular}

The states $1^{m_{a}}, m_{a}=0,1,2$, correspond to the totally symmetric skeletal vibration excited with 0,1 , and 2 quanta, respectively: $6^{1}$ denotes the quantum of $e_{2 g}$ C-bending motion on which the CC $\left(Q_{1 g}\right)$ progression is built. $(1 / \tau)=\left(1 / \tau_{r r}+1 / \tau_{r}\right)$, where $\tau_{r}$ is the pure radiative lifetime and $\tau_{n r}$ is the radiationless lifetime discussed in the text.

Further experimental work on benzene is presently in progress and is expected to provide more information on the effect of vibrational excitation 
on electronic relaxation processes. The Boltzmann counting method described above can be improved ${ }^{29}$ by taking into account the contribution of the vibrational overlaps in the neighbourhood of the maximum term. These corrections give rise to the proper pre-exponential factors in the energy gap form of the rate constant. The theory is also extended ${ }^{29}$ to allow for frequency changes, and to include the role of other stretching motions, and out-of-plane bends, as accepting modes. These considerations, in conjunction with the more extensive experimental data, allow for a revealing test of our understanding of the energy dependence of the non-radiative relaxation processes.

\section{ASPECTS OF THE THEORY OF PHOTOCHEMICAL REACTIONS}

A photochemical reaction differs from a thermal reaction in at least one fundamental way: the absorption of a photon by a molecule 'prepares' the molecule in a well defined nonstationary state with a nonuniform distribution of energy, whereas a thermally excited molecule is almost always in a state in which the distribution of energy over possible modes of motion is uniform, or nearly so. Thus, in the theory of thermal unimolecular reactions the rate of decomposition is relative to two factors:

(i) The collisional energy transfer leading to activation and,

(ii) The breaking of a particular bond.

It is usually assumed that the collisions are so 'strong' that the initial and final total energies are uncorrelated so that the probability that a particular molecule will have an energy $E$ after a collision is determined solely by the Boltzmann factor $g_{E} \exp (-E / k T)$, with $g_{E}$ the degeneracy of the state with energy $E$. It is also assumed that the coupling between the internal degrees of freedom is so strong that the consequent energy rearrangement can be described statistically, i.e. the probability that a particular molecule, with given total energy, will have a specified configuration after an internal energy exchange is calculated by describing the system in terms of the appropriate microcanonical ensemble. Finally, the bond which breaks is designated $a$ posteriori, and is not prescribed by theoretical argumentation.

Our interest in the theory of photochemical reactions started with the observation that in a molecule 'prepared' in a state with nonuniform distribution of the energy, the details of internal nuclear and electron dynamics should influence the rate and the products of the reaction. Now, as argued in earlier sections, in the upper excited states of a molecule it is often found that the vibrational levels corresponding to one Born-Oppenheimer (BO) manifold overlap, the dense set of vibrational levels corresponding to a lower electronic Born-Oppenheimer state. If the energy separation between the overlapping levels is comparable with, or less than, the off-diagonal matrix elements of the nuclear kinetic energy operator in the basis of BO states, the BO representation of the states of the molecule is no longer adequate. In this case the true molecular eigenstates can be thought of as mixtures of BO states. We have referred above to vibrational levels. In a highly excited molecule the harmonic approximation to the description of nuclear motion frequently is not adequate. We shall briefly return to this latter problem later: however, it is important to recognize at the outset that the theories which are familiar and satisfactory 


\section{RELAXATION PHENOMENA IN EXCITED MOLECULES}

for the description of the ground state and the lower excited states of a molecule must be extended, or even replaced, when what is sought is a description of the upper excited states of a molecule.

Consider, as a first example, a molecule with an energy spectrum of the type shown in Figures 2 and 3. This energy level scheme can be thought of as corresponding to the existence of a localized electronic excitation coupled to electronic ground state vibrations, which in turn are coupled to a fragmentation continuum. Examples of energy spectra resembling this are found in

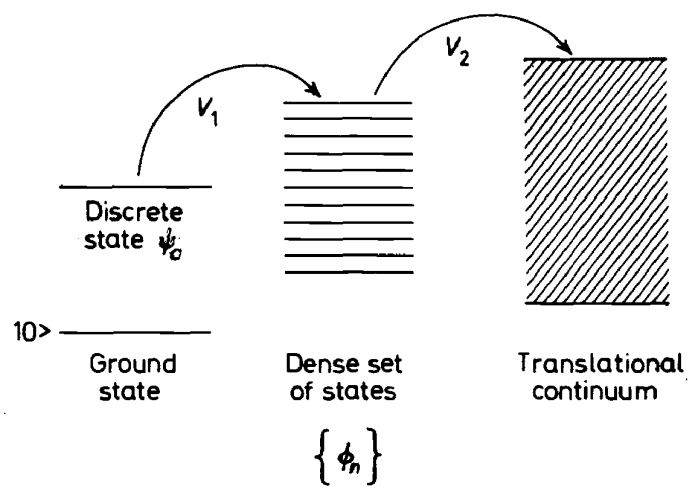

(a) Zero order states

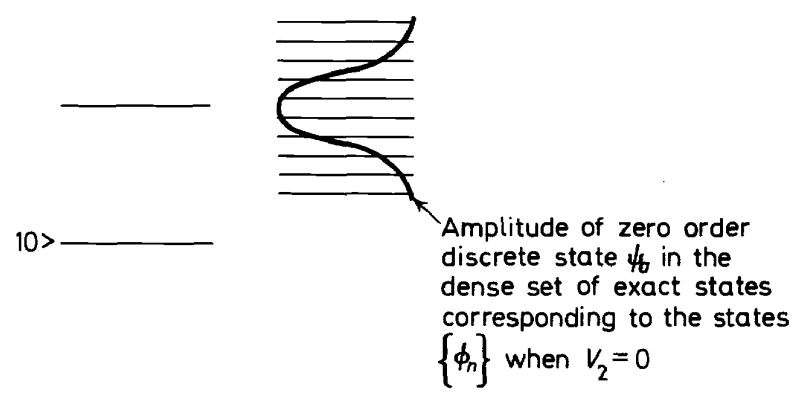

(b) Exact eigenstates in the absence of coupling to the continuum

Figure 2. Schematic energy-level diagram of (a) the zero-order states and (b) the exact eigenstates in the absence of coupling to the continuum.

many carbonyl compounds and in methyl substituted benzenes. In both classes of compounds localized excitation of a chromophore may lead to fragmentation at a distant chemical bond. ${ }^{30}$

The properties of the dissociative states of a molecule with the spectrum described above were studied by Rice, McLaughlin and Jortner ${ }^{31}$. They represented the true eigenstates of the molecule in the absence of a radiation 


\section{STUART A. RICE AND WILLIAM M. GELBART}

field as a superposition of zero order states (which need not be BO states but which are the eigenstates of a zero order separable Hamiltonian). Suppose that, as shown in Figure 3, transitions from the ground state to the dissociative continuum and to the dense manifold of bound zero order vibronic states are forbidden, but that to one discrete zero-order state is

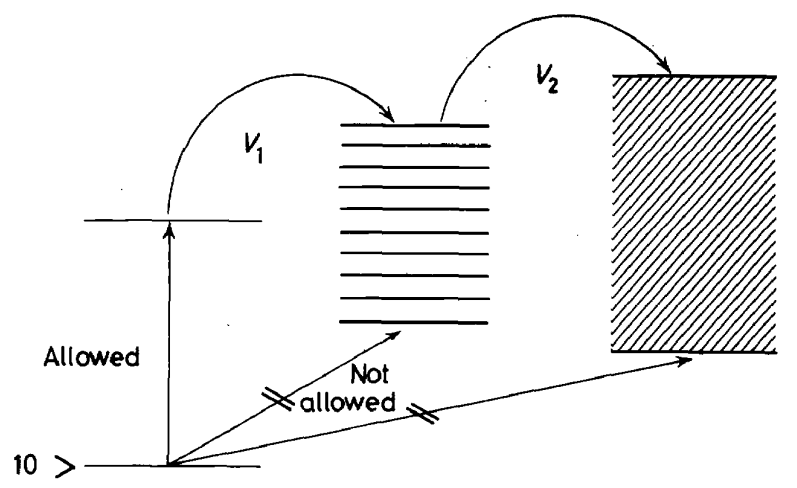

(a) Allowed dipole transitions between zero order states.

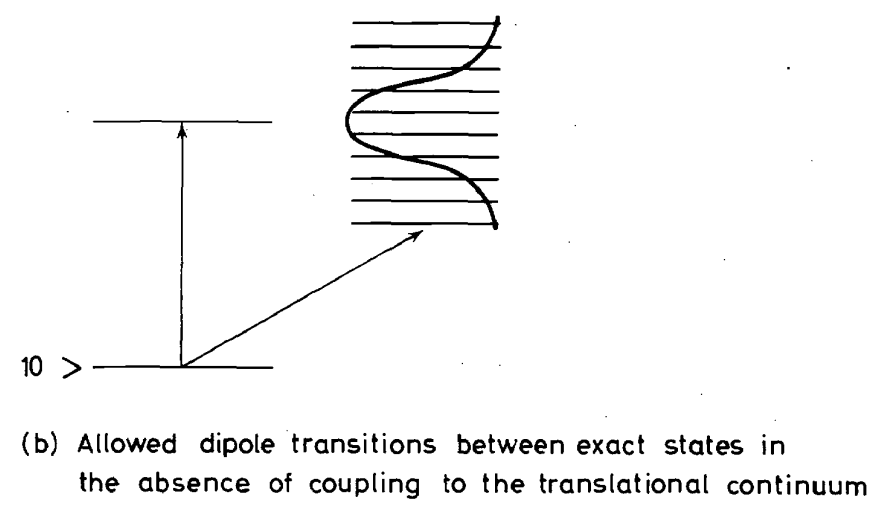

Figure 3. Schematic diagram showing transitions between the sets of levels of the hypothetical spectrum. (a) Allowed dipole transitions between zero-order states. (b) Allowed dipole transitions between exact states in the absence of coupling to the translational continuum.

allowed. Then absorption of a photon prepares the molecule in a nonstationary state which can be described as a time dependent superposition of exact eigenstates, each of which is itself a time independent superposition of zero order (say BO) states ${ }^{32}$. To study photodecomposition it is imagined that the molecule is illuminated with a pulse of radiation, and the time evolution of the nonstationary state is followed. One of the interesting features of this description is the dependence of the probability of the molecule being in a 
given nonstationary state on the time correlations in the coupled radiation field. Finally, the probability of dissociation of the molecule is related, formally, to the matrix elements coupling the component manifolds of the spectrum, and the densities of states in the dissociative continuum and the manifold to which it is connected.

It is possible, using an approximation relating to the influence of the rate of decomposition on the distribution of amplitude of the discrete zero order state in the dense set of exact vibrational states, to derive a simple expression for the decomposition rate. That expression is

$$
k=\frac{2 \rho_{2} v_{2}^{2}}{\hbar}\left[\frac{\pi}{2}-\tan ^{-1}\left(\frac{E_{m}-E_{a}}{\pi\left(\rho_{1} v_{1}^{2}\right)}\right)\right]
$$

where $E_{m}$ is the minimum energy required for fragmentation in the selected bond, $\rho_{1}$ and $\rho_{2}$ are the densities of states in the vibrational quasicontinuum and the fragmentation continuum, and $v_{1}$ and $v_{2}$ are the matrix elements connecting the discrete zero order state to the zero order vibrational quasicontinuum, and the zero order vibrational quasicontinuum to the fragmentation continuum, respectively. The limit $\rho_{1} v_{1}^{2} \ll \rho_{2} v_{2}^{2}$ corresponds to the case where the molecule dissociates as rapidly as the nonstationary wave packet can disperse into the vibrational quasicontinuum. This case is analogous to but not identical with that considered by Peters ${ }^{33}$ - direct excitation to a nonbonding state without intermediate intramolecular energy transfer. In the limit $\rho_{1} v_{1}^{2} \gg \rho_{2} v_{2}^{2}$ the rate of fragmentation is controlled by the rate of internal energy transfer.

Of what use is such a formal description? Can we learn from it anything not already known? We believe the answer to both these questions to be a qualified yes. Perhaps most important is the change in viewpoint put forward from that embodied in the classicial theory of unimolecular reactions (thermal or photochemical). In the current theory the excited molecule about to dissociate is regarded as being in a resonant scattering state ${ }^{34}$, and not a bound state. The process by which the molecule decomposes is then just a form of predissociation. Because the exact resonant scattering state is represented as a linear combination of the zero-order localized state, the dense set of zero order vibrational states, and the zero order continuum, and because the zero order state, which can be excited by absorption of a photon, is spread over many exact resonant scattering states, absorption of a photon leads to the population of resonant scattering states spread over a range of energy. Predissociation occurs from this band of states, subject to appropriate selection rules. Thus, it is not necessary to ascribe to the activated molecule the properties of a stable molecule, and the difficulties associated with the concepts of thermodynamic functions of the activated state, and the special role of the reaction coordinate, can be bypassed. Furthermore, by realizing that an excited molecule is in a resonant scattering state, the lifetime against decay into fragments appears naturally in the evaluation of the probability of dissociation-it need not be introduced as an ad hoc hypothesis defining irreversibility.

At a more detailed level the analysis raises the possibility that under suit- 
able excitation conditions a coherently excited set of resonant scattering states can interfere ${ }^{34}$, perhaps with consequences of chemical interest. Also, depending on the magnitudes of the several coupling matrix elements and densities of states the excited molecule in the resonant scattering state may behave like a long-lived vibrationally hot molecule, like a molecule that decomposes directly to fragments without internal energy transfer, or in an intermediate fashion not like either of the cited classical limits:

The model of the photodissociation reaction described above has been used by Heller ${ }^{35}$ to elucidate the nature of hydrogen transfer and hydrogen abstraction reactions. Suppose that, starting from an initial nonuniform distribution of energy in the excited state of the molecule, electronic-vibrational and vibrational-vibrational coupling leads to the excitation of particular vibrations. It is known that high frequency anharmonic stretching vibrations are the preferred energy acceptors in molecule conserving, radiationless process. Then the abundance of hydrogen abstraction and transfer reactions of electronically excited carbonyls and alkanes is explained by preferential energy transfer to high frequency stretching vibrations involving hydrogen. So long as the rate of vibrational deexcitation of such modes is small, relative to the rate of dissociation, the quantum yield of dissociated products should be large. The following rules are natural consequences of the properties built into the model considered ${ }^{35}$ :

(i) The most reactive atoms in a molecule are those that are involved in high frequency molecular motions,

(ii) Amongst bonds with nearly the same vibrational frequency the more anharmonic the vibration and the lower the bond energy the greater is the bond reactivity, and

(iii) Amongst bonds with nearly the same vibrational frequency and dissociation energy the closer the bond to the initial concentration of excitation energy the greater is the bond reactivity.

Assuming that there are no changes in relative bond strengths in the excited states of a molecule (as compared to the bond strengths in the ground state) the above model and rules, derived therefrom, correlate the observed intramolecular abstraction of hydrogen by excited carbonyl compounds (in both singlet and triplet states) and the $\mathrm{Hg}\left({ }^{3} P_{1}\right)$ sensitized fragmentation of alkanes. Of course in some molecules there are changes in relative bond strengths in the excited state relative to the gound state; the above rules do not apply to the photochemistry of these compounds.

Further evidence of the potential value of representing a unimolecular reaction in terms of the time evolution of a resonant scattering state can be glimpsed from the as yet, incomplete theory of photoisomerization ${ }^{36,25}$. Taking the cis-trans isomerization of stilbene as a prototype reaction Gelbart and Rice ${ }^{36}$ studied the properties of a model molecule in which a singlet vibronic level interacts (through spin-orbit coupling) with a nearly degenerate triplet state, which in turn is coupled (by vibronic interactions) to an isoenergetic set of closely spaced vibrational levels belonging to a lower triplet (see Figure 4). Unlike the photodissociation reaction, the cis-trans isomerization does not involve a true continuum since, even in the limit of free internal rotation, the moments of inertia of the molecular 'ends' may be sufficiently small to lead to internal rotational spacings not grossly different from those 


\section{RELAXATION PHENOMENA IN EXCITED MOLECULES}

characteristic of hindered torsional motion. If the molecule is large enough to be in the limit where $\rho v^{2} \geqslant 1$, where $\rho$ is the density of states in some manifold and $v$ the matrix element coupling that manifold to a discrete state, and this condition holds for all discrete states and dense manifolds of interest, then it is shown that the radiative and nonradiative processes proceed independently of one another. The fluorescence quantum yield and the emis-

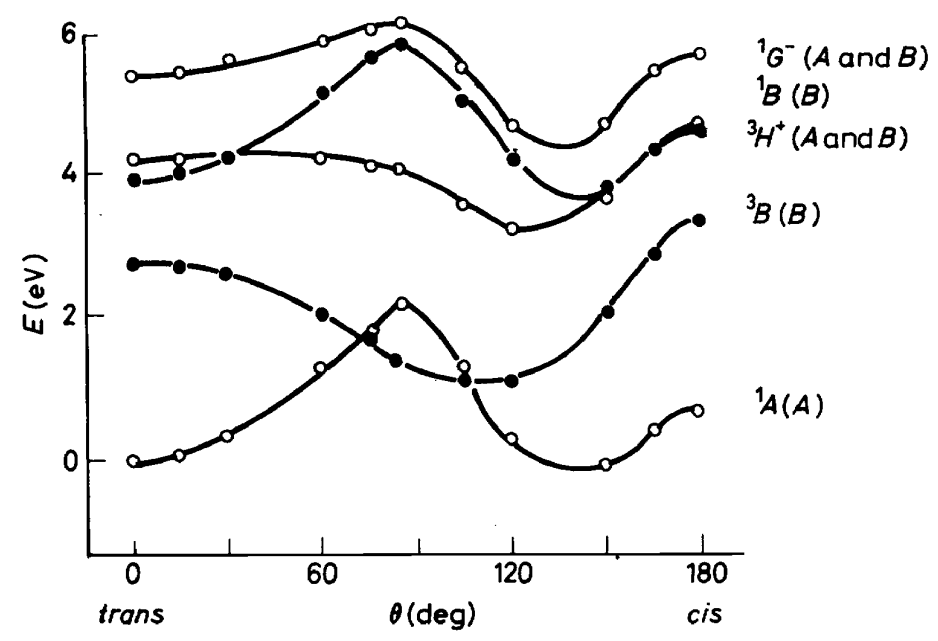

Figure 4. A schematic plot of electronic energy vs. angle of twist (about the double bond) in unsubstituted stilbene [from the SCF-CI calculations of P. Borrell and H. H. Greenwood, Proc. Roy. Soc. (London) A298, 453 (1967)].

sion lifetime predicted by this model depend explicitly on the (solvent specific) energy difference between the discrete singlet and the triplet, and they may be conveniently parameterized in terms of the level density and coupling matrix elements of the zero-order states.

The role of hindered rotation in the breakdown of the BO approximation can, in fact, be analyzed in considerable detail ${ }^{25}$. It can be shown that the molecular mode whose equilibrium positions differ greatly in the initial and final electronic states (the strongly coupled degree of freedom) may be separated out from the other vibrations in the general expression for the rate of reactions. It is then found that, under conditions appropriate to the description of cis-trans isomerization in stilbene-like molecules, most of the electronic energy change should go into the torsional degree of freedom rather than the usual vibrational modes which serve as acceptors if all modes are weakly coupled. The experimentally observable consequence of this conclusion is the lack of (or great diminution of) an isotope effect in the deactivation of the lowest triplet to yield the isomer product. Sufficient experimental data are not now available to test these predictions.

In the classical theory of unimolecular reactions and in the theory of photochemical reactions recently developed the internal transfer of energy 
plays an important role. Nevertheless the mechanism of the internal energy transfer and its relationship to specific reaction product selection is not understood. Some insight into the energy transfer process and its coupling to molecular dissociation can be obtained from the study of models. Gelbart, Rice and Freed ${ }^{37}$ have examined the properties of a model in which all vibrations are coupled, some weakly and some strongly, to all others, and in which all bonds may break with specified dissociation probabilities. The model is sufficiently general that the nature of the vibrations need not be specified. Thus it is, in principle, possible to account for the breakdown of the normal mode-harmonic oscillator description of a highly excited moleculeharmonic oscillator description of a highly excited molecule-although only in a phenomenological fashion. A stochastic process defined by a set of transition probabilities, which allows one to explicitly include the dynamics of the inter- and intra-molecular energy exchange, replaces the usual microcanonical ensemble and strong collision assumptions. By providing for the breaking of all bonds at all times and including the competing effects of energy transfer and bond dissociation, information can be obtained about which bond is most likely to break. The following cases of interest have been studied:

(i) If the distribution of energy in the initial state is uniform, corresponding to collisional activation of a gas of polyatomic molecules originally in thermal equilibrium, it is shown that upon 'heating up' the gas, all other things being equal (viz. all bond energies the same), the probabilities for the breaking of the individual bonds are equal at all times, regardless of the details of the vibrational interactions. This result is rendered plausible when it is noted that at sufficiently high pressures the rate of collisional excitation exceeds the rate of internal energy transfer. The lack of great specificity in collisional excitation results, in a collection of molecules, in almost equal probability of excitation of all degrees of freedom. Then the details of the vibrational interactions are clearly unimportant and the dissociation probabilities depend on the internal energy distribution. At very low pressures the reaction rate is determined by the rate of collisional activation. Thus, except for a small range of pressure where the rate of collisional excitation is comparable with the rate of internal energy transfer, it is to be expected that individual bond dissociation probabilities will be sensibly independent of the nature of the internal coupling.

The result, just cited, provides a formal justification of the assumption implicit in classical unimolecular reaction rate theory, namely that the reaction rate depends only on the equilibrium internal energy distribution of the activated molecule.

(ii) If the distribution of energy in the initial state is non-uniform, corresponding to certain kinds of photochemical, hot atom, or photosensitization excitation, it is found that, all other things being equal (again, similar dissociation strengths), the more strongly coupled vibrational motions will always be more likely to lead to fragmentation of the molecule than is the case for the weakly coupled vibrations. Furthermore, by virtue of the energy flow into strongly coupled degrees of freedom, a strong bond can break before a weak bond if that weak bond is more weakly coupled to the site where the energy is initially localized. 


\section{RELAXATION PHENOMENA IN EXCITED MOLECULES}

The result, just cited, is consistent with the rules deduced by Heller ${ }^{35}$, with the competing fragmentation reactions observed in the mass spectrometric studies of Rosenstock ${ }^{38}$ et al. and with the chemical activation studies of Rabinovitch and coworkers ${ }^{39}$; in all of these cases there are formed excited molecules, molecular ions and/or radicals with strongly non-uniform internal energy distributions.

(iii) In case (ii) each internal degree of freedom can exchange energy with every other degree of freedom. If, instead, interactions are restricted to 'nearest neighbours', so that a linear chain of interactions exists, different results are obtained. As can be inferred from the work of Magee and coworkers ${ }^{40}$, in a linear chain of bonds, one of which is weakly coupled, it is the weakly coupled bond which breaks because the mean time which the energy spends localized in any of the individual bonds depends inversely on the strength of coupling of that bond to its neighbours. The weakly coupled bond then 'traps' the otherwise delocalized energy for the longest time and is therefore the most likely to break, regardless of the dissociation mechanism. The nature of the competition between energy transfer and fragmentation thus depends in a fundamental way on whether the molecule in question can be regarded as 'effectively linear', is intrinsically nonlinear, or is intermediate between these two limits.

Related to the linear chain calculations of Magee et al. is the molecular exciton model which Partridge has recently postulated as an explanation of electronic excitation transfer in alkane polymers ${ }^{41}$. The $\mathrm{CH}$ and $\mathrm{CC}$ bonds of, say, polyethylene are treated as separate entities (i.e. electron exchange is neglected) which interact through nearest- and next-nearest neighbour transition dipoles during excitation. In this way the principal intramolecular interactions dominate the interchain ones: the theory predicts that there will be sensibly no change in the electronic absorption spectra of these polymers as they pass from the gas to solid phase or as they change their conformation. The lowest edge of the lowest band is found to be a pure $\mathrm{CC}$ bond exciton level, but with increasing energy $(K)$ some $\mathrm{CH}$ bond excitation begins to be mixed in. The narrow upper band is a pure $\mathrm{CH}$ exciton band in which there is no excitation at all of the CC bonds. In fact, in the nearest-neighbour approximation the exciton interaction is wholly within pairs of $\mathrm{CH}$ bonds attached to the same carbon atom (with no coupling between these pairs and hence degeneracy of the energy levels); in the next-nearest-neighbour description the different pairs interact slightly and there is a small splitting of levels. Thus if a certain $\mathrm{CH}$ bond is excited the energy will primarily be shared between this bond and its other $\mathrm{CH}$ bond neighbour on the same carbon atom-only occasionally will it migrate to other $\mathrm{CH}$ bonds. This exciton mode will consequently give rise to little energy transfer down the polymer chain and, with the excitation essentially localized in just two $\mathrm{CH}$ bonds, it is very probable that one or both of them will break. $\mathrm{H}$ atom and $\mathrm{H}_{2}$ molecule elimination from long chain alkanes is in fact observed commonly under vacuum-uv irradiation ${ }^{41}$. The $\mathrm{CC}$ exciton band, on the other hand, involves a sharing of excitation between all of the $\mathrm{CC}$ bonds. The model therefore predicts that the probability of a main chain (CC bond) scission should be very small since the energy is not localized in any one bond long enough to break that bond. Energy transfer is also possible to chemical defects or to 
foreign atoms and molecules lying close by, but not directly attached to, the polymer molecules.

This description of Partridge's certainly constitutes a beginning step in a proper understanding of the radiation chemistry kinetics of long chain saturated molecules. It is important to note, however, that he has not considered at all the effect of electronic-nuclear coupling and the very probable importance of vibrational relaxation in determining the excitation energy transfer.

\section{RELEVANT EXPERIMENTS AND EMPIRICAL APPROACHES}

We have seen that one must take into explicit account the competing effects of the vibrational interactions in order to properly determine the kinetics of unimolecular reactions. At the same time one is confronted with the fact that hardly anything at all is known about these quantities, i.e. one is almost wholly ignorant of the relevant properties of the nuclear potential energy surface in the region of large displacements from equilibrium. Nevertheless, it is possible to formulate a set of semi-empirical rules which allow one to extrapolate back and forth between the dissociation limit and the limit of small vibrational quantum numbers. It is of interest, then, to consider various schemes of this kind, as well as new experiments, which can provide preliminary information on the actual dynamics of intramolecular energy exchange and dissociation.

A most interesting example of the semi-empirical approach is provided by the work of Bader ${ }^{42}, \mathrm{Salem}^{43}$, and Pearson ${ }^{44}$, and their coworkers. These investigators have studied the way in which the electron density in a molecule is allowed to follow the nuclear vibrations by taking into account corrections to the crude adiabatic approximation. In this way they are able to formulate certain conditions which relate the most probable unimolecular reaction coordinate to the symmetries of the normal mode motions and the nature of the low-lying electronic states. For, given small enough displacements, one can rigorously show (to second order in perturbation theory) that the electronic energy of a distorted configuration in the $i^{\text {th }}$ normal coordinate, $Q_{\dot{b}}$ is given by

$$
E\left(Q_{i}\right)=E(0)+\left\{\left\langle\psi_{0}\left|\left(\frac{\partial^{2} U}{\partial Q_{i}^{2}}\right)_{0}\right| \psi_{0}\right\rangle-\sum_{k \neq 0} \frac{\left|\left\langle\psi_{0}\left|\left(\partial U / \partial Q_{j}\right)_{0}\right| \psi_{k}\right\rangle\right|^{2}}{E_{k}-E_{0}}\right\} Q_{i}^{2},
$$

where $U$ is the electron-nuclear coulomb energy and $\left\{E_{k}\right\}$ and $\left\{\psi_{k}\right\}$ are the energies and wavefunctions corresponding to the zero-order electronic states at the equilibrium configuration. The first term in $Q_{i}^{2}$ gives the increase in energy of the system when the nuclei are displaced by $Q_{i}$ and the electron distribution is held fixed according to $\psi_{0}$. The matrix elements in the sum of the second term mix the ground and excited states and thereby allow the electron distribution to relax (follow the motion of the nuclei), hence lowering the energy. Accordingly, the energy is lowered the most for that $Q_{i}$ which mixes in the first excited state. It is then assumed that this lowering obtains for larger displacements as well $\left[\mathrm{Salem}^{43}\right.$ however, warns of the possible dangers involved here, and argues the importance of the cubic potential 


\section{RELAXATION PHENOMENA IN EXCITED MOLECULES}

contributions]. Predictions of the most probable unimolecular reaction coordinate follow immediately: the actual results seem to work surprisingly well even though the rules are implicitly based on an extrapolation from the region of small amplitude vibrations to the dissociation limit. From our present point of view there is still a more important conceptual failingnamely, the nuclear potential energy is taken to be separable with respect to some set of internal coordinates. That is, it is still only the diagonal anharmonicities which are included - the effect of the off-diagonal vibrational interactions is neglected. An extension taking into account these couplings would constitute an important step in developing a new set of correlation rules for the a priori prediction of reaction coordinates.

We should mention here the sensitization experiments in which mercury atoms, excited to their metastable singlet and triplet states, are allowed to collide with polyatomic molecules of interest, thereby preparing them in high-lying vibrational levels of the ground electronic state. This class of reactions is of special interest because it provides the cleanest examples of a fragmentation process in which the weakest bonds are not always the most likely to break. Heller ${ }^{35}$ has considered in particular the mercury $\left({ }^{3} P_{1}\right)$ sensitized photolysis of alkanes, and observes that the most reactive bonds are not the CC-sigma bonds but the stronger (by as much as $20 \mathrm{kcal} / \mathrm{mole}$ ) $\mathrm{CH}$ bonds. In order to explain this behaviour he assumes that the efficiency of energy transfer from the excited mercury to the internal degrees of freedom of the alkane is directly governed by the appropriate Franck-Condon overlap integrals. It follows immediately that the best accepting vibrations will be those of highest frequency and anharmonicity and therefore that the hydrogen stretching motions will be preferentially excited. It is not at all obvious, however, that the atom-molecule energy transfer can be described by such a simple assumption. Rather it seems likely that more complicated vibrational motions of the alkane are initially excited and that the reactivity of the $\mathrm{CH}$ bonds needs to be explained by a different mechanism. Our contention could be investigated by an experiment which measures the lifetime of the sensitized molecule against fragmentation. If this time lag were long, compared with a $\mathrm{CH}$ vibrational period, one could infer that the hydrogen atoms are not directly activated by sensitization, but rather are preferentially excited by intramolecular energy transfer according to the stochastic theory of Gelbart, Rice, and Freed ${ }^{37}$ described in IV.

A more fruitful class of experiments could be modelled on certain studies of Frey $^{45}$ Kistiakowsky $^{46}$ and Rabinovitch ${ }^{47}$, which were intended to test, 'unambiguously', the fundamental strong coupling assumption of unimolecular reaction theory. In 1960, Frey ${ }^{45}$ presented interesting evidence suggesting that the energy exchange between normal mode motions might in fact occur slowly compared with the lifetime of the excited molecule. He studied the addition of methylene to isobutene, and the subsequent isomerization of the vibrationally excited 1, 1-dimethylcyclopropane into 2-methylbut-2-ene (I) and 3-methylbut-1-ene (II). It was found that the ratio of the yields of products I and II is roughly 1.60 whereas the corresponding value obtained in the thermal isomerization of 1,1-dimethylcyclopropane is as low as 0.96 This result suggests that the initial distribution of vibrational energy in the addition reaction is non-uniform and that the rearrangement 
of the 'hot' molecule occurs before the randomization, implied by the strong coupling theories, can take place.

Butler and Kistiakowsky ${ }^{46}$ have investigated the validity of these assumptions in a different, and yet related, experiment. They studied the isomerization of vibrationally excited methylcyclopropane prepared by the addition of methylene to cyclopropane, and to propylene. It was found that the yields of the various butene products obtained from the cyclopropane addition are within experimental error of those found from the propylene reaction and that these values are in turn the same as those determined in studies of the direct thermal isomerization of methylcyclopropane. This lack of dependence of the several reaction rates on the initial vibrational energy distributions, in contrast to the earlier conclusions of Frey, was taken to confirm the applicability of the strong coupling assumption. We feel, however, that all these experiments, while properly conceived in spirit, are undermined by the effect of collisions. That is, since the isomerization and dissociation lifetimes are greater than the mean time between encounters in the gas, the internal energy distribution must surely be disturbed by collisions before unimolecular reaction occurs. In order to obtain information on the intramolecular dynamics unambiguously, then, it would be necessary to repeat these experiments at total pressures which are lower by several orders of magnitude. Still better, one can look to chemical lasers and crossed molecular beams for the least ambiguous resolution of these questions.

The relevant chemical laser experiments of special interest are the bimolecular $^{48}$ and photo-elimination ${ }^{49}$ reactions investigated by Pimentel and his co-workers. In the former case, one studies exothermic exchange processes of the type $A+B C \stackrel{k_{v}}{\rightarrow} A B_{v}^{*}+C:$ in particular, say, the reaction $\mathrm{F}+\mathrm{H}_{2}\left(\mathrm{D}_{2}\right) \rightarrow \mathrm{HF}_{v}^{*}\left(\mathrm{DF}_{v}^{*}\right)+\mathrm{H}$, which arises in the flash photolysis initiated $\mathrm{UF}_{6}-\mathrm{H}_{2}\left(\mathrm{D}_{2}\right)$ chemical lasers. By measuring the vibrational energy distribution of the diatomic products, and invoking microscopic reversibility arguments, these experiments bear directly on our understanding of the role of internal excitation in simple collision dynamics. At the same time, by studying, say, other HF(DF) chemical lasers [e.g. those arising from $H(D)$ atom abstractions in the alkanes] one can begin to learn about the effect of exothermicity of reaction on the distribution of vibrational energy amongst the products.

Perhaps of more interest are the photoelimination ${ }^{49}$ chemical lasers developed by Berry and Pimentel. They have studied in detail the $\mathrm{HCl}$ emission following vacuum uv photolysis of the three dichloroethylenes. These experiments point up the way in which the vibrational excitation of the $\mathrm{HCl}$ produced by photoelimination depends upon the structure of the parent molecule. By microscopic reversibility it is inferred that in the reverse (addition) reaction, $\mathrm{HCl}$ vibrational excitation will show a stereospecific preference for the 11 - over the 1,2-dichloroethylene product, and further for the cis-1,2- over the trans-1,2- isomer.

The final, and what we believe to be the most fruitful, class of experiments relevant to this discussion is the crossed molecular beam method which has been developed over the past fifteen years by several groups of investigators. Of special interest are the chemical reactions which proceed through the formation of a collision complex with a lifetime much longer than a rotational 
period. Here the angular distribution of products peaks symmetrically forward and backward in the centre-of-mass system. ${ }^{50}$ Several examples of this behaviour have been reported in the recent molecular beam literature, most notably for the alkali-alkali halide ${ }^{51}$ and alkali halide-alkali halide exchange reactions ${ }^{52}$. These studies are of importance to us here insofar as they allow for comparisons between the various 'competing' theories of unimolecular dissociation. The distribution of total energy amongst the vibrations and rotations of the products, and the relative motion of the separating fragments. can be estimated experimentally by combining the data from velocity analyses and angular scans. In turn these detailed results can be calculated by a modified RRKM-transition state theory ${ }^{51,52}$ or by the phase space approach of Light ${ }^{53}$. Rough agreement is found ${ }^{51}$ in both cases and is taken to confirm the validity of the statistical assumption for these relatively long-lived collision complexes.

These examples, almost by definition, do not lend themselves to a weak coupling stochastic theory of the form considered by Gelbart, Rice and Freed $^{37}$. During the last year, however, work has begun at the University of Chicago on cross-beam experiments which are of a decidedly different nature. It is planned ${ }^{54}$ to study the product and energy distributions arising from the addition of fluorine atoms to various ethylene derivatives. Preliminary data from angular scans of the reaction $\mathrm{F}+\mathrm{C}_{2} \mathrm{H}_{4} \rightarrow \mathrm{C}_{2} \mathrm{H}_{4} \mathrm{~F}^{*} \rightarrow$ $\mathrm{C}_{2} \mathrm{H}_{3} \mathrm{~F}+\mathrm{H}$ show a symmetric forward-backward peaking, indicating a long lived $\left(\tau_{c}>10^{-12} \mathrm{sec}\right)$ collision complex. Rough calculations already suggest that a considerably larger fraction of the available total energy is coupled into the relative motion of the separating fragments than is consistent with the statistical theories. Velocity analysis of the products will allow for more dependable estimates of the most probable distribution of translational and rotational energy in the polyatomic fragment. It will be of interest to run the crossed beam experiment, as well, for addition reactions involving longer chain alkenes, particularly those having weak bonds at the far end of the molecule from the ethylenic linkage. The species of products, their angular distribution and the energy of the relative motion of the separating fragments will indicate the extent of vibrational relaxation which has taken place before dissociation. This kind of information, aside from generally confirming the need for a full stochastic description of the internal energy dynamics, should also provide some preliminary knowledge of the 'off-diagonal' vibrational interactions in highly excited molecules.

\section{ACKNOWLEDGEMENTS}

Our research in photochemistry has been supported by the Directorate of Chemical Sciences. Air Force Office of Scientific Research. We have also benefited from the use of facilities provided by the Advanced Research Projects Agency for materials research at the University of Chicago.

\section{REFERENCES}

1 The reader is referred to the review article by J. Jortner, S. A. Rice and R. M. Hochstrasser, Advan. Photochem. 7, 149 (1969), for a discussion of many of the mathematical details omitted in this paper. 
${ }^{2}$ G. W. Robinson and R. P. Frosch. J. Chem. Phys. 38, 1187 (1963);

37, 1962 (1962).

3 M. Bixon and J. Jortner. J. Chem. Phys. 48, 715 (1968).

4 W. Rhodes, B. Henry and M. Kasha. Proc. Nat. Acad. Sci. U.S. 63, 31 (1969).

5 P. Pringsheim. Fluorescence and Phosphorescence, p. 271. Interscience: New York (1949).

${ }^{6}$ R. J. Watts and S. J. Strickler. J. Chem. Phys. 44, 2423 (1966).

7 R. Williams and G. J. Goldsmith. J. Chem. Phys. 39, 2008 (1963).

8 Perylene: W. R. Ware and P. T. Cunningham. J. Chem. Phys. 44, 4364 (1966).

9 Anthracene: K. H. Hardetl and A. Scharmann. Z. Naturforsch. 12a, 715 (1957).

10 G. B. Kistiakowsky and C. S. Parmenter. J. Chem. Phys. 42, 2942 (1965).

11 U. Fano. Phys. Rev. 124, 1866 (1961).

12 D. Chock, J. Jortner, and S. A. Rice. J. Chem. Phys. 49, 610 (1968).

13 J. Jortner and R. S. Berry. J. Chem. Phys. 48, 2757 (1968).

14 K. F. Freed and J. Jortner. J. Chem. Phys. 50, 2916 (1969).

15 M. Bixon, J. Jortner and Y. Dothan. Mol. Phys. 17, 109 (1969); see also M. Bixon and J. Jortner. J. Chem. Phys. 50, 4061 (1969).

16 K. F. Freed. J. Chem. Phys. 52, 1345 (1970).

17 M. Bixon and J. Jortner. J. Chem. Phys. 50, 3284 (1969).

18 A. E. Douglas. J. Chem. Phys. 45, 1007 (1966), and references cited therein.

19 See W. Siebrand. J. Chem. Phys. 47, 2411 (1967) and earlier papers.

20 See J. P. Byrne, E. F. McCoy and I. G. Ross. Australian J. Chem. 18, 1589 (1965) and earlier papers.

21 An excellent review of these solid-state problems is given by J. J. Markham. Rev. Mod. Phys. 31, $956(1959)$.

22 R. Kubo. Phys. Rev. 86, 929 (1952).

${ }^{23}$ S. H. Lin. J. Chem. Phys. 44, 3759 (1966); S. H. Lin and R. Bersohn, ibid. 48, 2732 (1968).

24 R. Englman and J. Jortner. Mol. Phys. 18, 145 (1970).

25 W. M. Gelbart, K. F. Freed and S. A. Rice. J. Chem. Phys. 52, 2460 (1970).

${ }^{26} \mathrm{~K}$. F. Freed and J. Jortner. J. Chem. Phys. in press.

27 W. M. Gelbart, K. G. Spears, K. F. Freed, J. Jortner and S. A. Rice. Submitted to Chem. Phys. Letters.

28 Fluorescence quantum yields are presently being determined in this laboratory by K. G. Spears and S. A. Rice, as well as by C. S. Parmenter (private communication).

29 W. M. Gelbart, Ph.D. thesis, University of Chicago (1970).

${ }^{30}$ See, for example, J. Calvert and J. Pitts. Photochemistry. John Wiley \& Sons: New York (1967); also P. Johnson and S. A. Rice. Chem. Phys. Letters 1, 709 (1968).

31 S. A. Rice, I. McLaughlin and J. Jortner. J. Chem. Phys. 49, 2756 (1968).

32 F. Mies and M. Krauss have used a related method in their elegant theory of unimolecular reactions. They explicitly employ a generalization of the Fano theory of autoionization but they have given less emphasis than we to internal energy redistribution since they study, primarily, thermally excited molecules. F. H. Mies and M. Krauss. J. Chem. Phys. 45, 4455 (1966); F. H. Mies. J. Chem. Phys. 51, 787, 798 (1969).

33 D. Peters. J. Chem. Phys. 41, 1046 (1964).

34 See also the work cited in reference 33.

35 A. Heller. Mol. Photochem. 1, 257 (1969).

36 W. Gelbart and S. A. Rice. J. Chem. Phys. 50, 4775 (1969).

37 W. Gelbart, S. A. Rice and K. Freed. J. Chem. Phys. in press.

${ }^{38}$ See, for example, H. Rosenstock, M. Wallenstein, A. Wahrhaftig and H. Eyring. Proc. Nat. Acad. Sci. U.S. 38, 667 (1952); also H. Rosenstock and M. Krauss. Mass Spectrometry of Organic Ions Ed. F. W. McLafferty (Academic Press, N.Y., 1963), Chapter 1.

39 C. W. Larson, B. S. Rabinovitch and D. C. Tardy. J. Chem. Phys. 47, 4570 (1967);

45, 1163 (1966); M. J. Pearson and B. S. Rabinovitch. J. Chem. Phys. 42, 1624 (1965);

B. S. Rabinovitch, R. F. Kubin and R. E. Harrington. J. Chem. Phys. 38, 405 (1963);

R. E. Harrington, B. S. Rabinovitch and R. W. Diesen. J. Chem. Phys. 32, 1245 (1960)

B. S. Rabinovitch and R. W. Diesen. J. Chem. Phys. 30, 735 (1959).

40 J. Magee and K. Funabashi. J. Chem. Phys. 34, 1715 (1961); J. C. Lorquet, S. G. El Komoss and J. Magee. J. Chem. Phys. 35, 1991 (1962).

41 R. H. Partridge. J. Chem. Phys. 52, 2485, 2491, 2501 (1970) and earlier papers.

42 R. F. W. Bader. Mol. Phys. 3, 137 (1960); Can. J. Chem. 40, 1164 (1962). 


\section{RELAXATION PHENOMENA IN EXCITED MOLECULES}

${ }^{43}$ L. Salem. Chemi Phys. Letters 3, 99 (1969); L. Salem and J. S. Wright. J. Am. Chem. Soc. 91 , 5947 (1969).

44 R. G. Pearson. J. Am. Chem. Soc. 91, 4947 (1969); J. Chem. Phys. 52, 2167 (1970).

${ }^{45}$ H. M. Frey. Trans. Faraday Soc. 56, 51 (1960).

46 J. N. Butler and G. B. Kistiakowsky. J. Am. Chem. Soc. 82, 759 (1960).

47 R. E. Harrington, B. S. Rabinovitch and H. M. Frey. J. Chem. Phys. 33, 1271 (1960).

48 J. H. Parker and G. C. Pimental. J. Chem. Phys. 51, 91 (1969); 48, 5273 (1968);

K. L. Kompa, J. H. Parker and G. C. Pimental. ibid. 49, 4257 (1968).

49 M. J. Berry and G. C. Pimental. J. Chem. Phys. in press; also ibid. 49, 5190 (1968) and 51, 2274 (1969).

50 D. R. Herschbach. Discussions Faraday Soc. 33, 149 (1962).

51 W. B. Miller, S. A. Safron and D. R. Herschbach. Discussions Faraday Soc. 44, 108 (1967).

52 W. B. Miller. Ph.D. Thesis, Harvard University (1969).

53 J. C. Light. Discussions Faraday Soc. 44, 14 (1967) and references to earlier papers cited therein.

54 Y. T. Lee and J. Parson. Private communication. 